Irish Math. Soc. Bulletin

Number 77, Summer 2016, 7-8

ISSN 0791-5578

\title{
The Structure of Spectrally Bounded Operators on Banach Algebras
}

\author{
MATTHEW YOUNG
}

This is an abstract of the $\mathrm{PhD}$ thesis The Structure of Spectrally Bounded Operators on Banach Algebras written by Dr. Matthew Young under the supervision of Dr. Martin Mathieu at the School of Mathematics and Physics, Queen's University Belfast, and submitted in May 2016.

Let $A$ and $B$ be unital Banach algebras and $T: A \rightarrow B$ a linear mapping. We say $T$ is spectrally bounded if there is $M>0$ such that $r(T x) \leq M r(x)$ for all $x \in A$, where $r(\cdot)$ denotes the spectral radius. If $r(T x)=r(x)$ for all $x \in A$, then $T$ is called a spectral isometry. My thesis is concerned with further understanding these operators.

Although the terms were introduced by Mathieu in [1], the history of these operators originates in Banach algebra and automatic continuity theory in the 1970s. In the latter, they are important as they provide the link between the analytic and algebraic structure of the Banach algebra [2].

Spectrally bounded operators are closely interconnected with the non-commutative Singer-Wermer conjecture, which is equivalent to asking the following question: if the commutator $[x, \delta x]$ belongs to the centre modulo the radical for all $x$ in a Banach algebra $A$ does this imply that the derivation $\delta$ is spectrally bounded? This is likely the deepest and most important open problem on spectrally bounded operators.

In [3], Mathieu proposed the following conjecture: every unital surjective spectral isometry between unital $C^{*}$-algebras is a Jordan isomorphism. See [4] and [5] for more details. We focus on furthering our understanding of this conjecture by placing ourselves in the setting of unital semisimple Banach algebras and consider a particular

2010 Mathematics Subject Classification. 47B48.

Key words and phrases. Spectrally bounded operators, spectral isometries.

Received on 20-5-2016.

Support from DEL is gratefully acknowledged. 
class of operators between them; the class of elementary operators. We establish that for arbitrary length elementary operators with given conditions, surjectivity of the operator is equivalent to the operator being an inner automorphism. Additionally, we show that a unital surjective spectrally bounded elementary operator of length three on a unital arbitrary $C^{*}$-algebra is a Jordan automorphism.

We also close some gaps in the literature for the situation for commutative Banach algebras. We examine the case where our spectral isometries are neither unital nor surjective. In general we lose multiplicativity, however using Gelfand theory and techniques from function space theory, we recover a multiplicative induced mapping into $C(X)$ where $X$ is a particular subset of the Gelfand space of the codomain.

\section{REFERENCES}

[1] M. Mathieu: Where to find the image of a derivation, Functional analysis and operator theory (Warsaw, 1992), Vol 30, Polish Acad. Sci., Warsaw, 1994.

[2] M. Mathieu: Interplay between spectrally bounded operators and complex analysis, Irish Math. Soc. Bull., (72) (2013) 57-70.

[3] M. Mathieu and G. Schick: First results on spectrally bounded operators, Studia Math., Vol 152, (2002) (2) 187-199.

[4] M. Mathieu: Towards a non-selfadjoint version of Kadison's theorem, Ann. Math. Inform., (32) (2005) 87-94.

[5] M. Mathieu: A collection of problems on spectrally bounded operators, AsianEur. J. Math., Vol 2, (2009) (3) 487-501.

(from May 2016) School of Mathematics And Physics, Queen's UniVERSITY BELFAST

E-mail address, Matthew Young: myoung14@qub.ac.uk 